\title{
Implementation Oriented Two-Sample Phase Locked Loop for Single-Phase PFCs
}

\begin{abstract}
A low-resource-consuming digital implementation of the Two-Sample (2S) Phase Locked Loop

(PLL) for low cost single-phase Power Factor Correction (PFC) converters is proposed. The design replaces trigonometric functions with an oscillator and divisions with approximations, without reducing the 2S-PLL synchronization capability. The proposal is evaluated and validated by simulation and experimentally.
\end{abstract}

\section{INTRODUCTION}

Low-cost Power Factor Correction (PFC) stages require simple and effective controllers to achieve the cost and performance targets [1], [2]. The 2S-PLL, proposed in [3], provides synchronization to the electrical grid while keeping relatively low the associated computational burden. However, the characteristics of the quadrature signal generator (QSG) in the 2S PLL make it difficult to use with low sampling rates, as it will be shown. This proposal modifies the 2S-PLL in [3] maintaining the synchronization performance while sampling at low sampling rates, typically at the power converter switching period.

\section{DIGITAL IMPLEMENTATION OF THE TWO-SAMPLE PLL}

The QSG proposed in [3] obtains the virtual in-quadrature component, $\beta$, by applying finite differences around an operation point, which can be dynamically adjusted as a function of the PLL frequency, $\omega$. Computational delays are compensated within the QSG. At instant $k, \beta_{k}$ is generated with only two samples of the grid voltage acquired in three consecutive sample instants:

$$
\beta_{k}=\left(\alpha_{k-2}-\alpha_{k}\right) \frac{1}{\sin \left(\frac{4 \pi}{N}\right)}+\alpha_{k} \tan \left(\frac{2 \pi}{N}\right)
$$




$$
N=\frac{2 \pi}{T_{s} \omega}
$$

where $\alpha_{k}$ is the sampled grid voltage, $T_{s}$ is the sampling time and $\omega$ is the grid frequency provided by the PLL. This approach minimizes the memory requirements of the QSG and keeps the orthogonality in the case of frequency variations by adjusting $\mathrm{N}$.

The trigonometric functions in (1) are simplified by applying Taylor series expansions, which are truncated at the second term, instead of the first one, due to the low sampling frequency approach, then (1) converts to

$$
\beta_{k}=\left(\alpha_{k-2}-\alpha_{k}\right) \frac{3 N^{3}}{12 \pi N^{2}-32 \pi^{3}}+\alpha_{k}\left(\frac{2 \pi}{N}+\frac{8}{3} \frac{\pi^{3}}{N^{3}}\right)
$$

which, applying the Taylor series expansion of $\frac{1}{1+x} \approx 1-x$, is rewritten as

$$
\beta_{k}=\left(\alpha_{k-2}-\alpha_{k}\right) K_{1}\left(1-K_{2} \Delta \omega\right)+\alpha_{k}\left(\omega T_{s}+\frac{1}{3}\left(\omega T_{s}\right)^{3}\right)
$$

where, $\Delta \omega$ is the frequency variation detected in the PLL with respect to the given nominal grid frequency, $\omega_{o},-$ the output of the PLL loop filter, typically a PI controller-, $K_{1}=\frac{1}{\left(2 T_{s} \omega_{0}-\frac{8}{6} T_{s}^{3} \omega_{0}^{3}\right)}$, $K_{2}=\frac{2-4 T_{s}^{2} \omega_{0}^{2}}{2 \omega_{0}-\frac{8}{6} T_{s}^{2} \omega_{0}^{3}} \cdot$ As a result, from (1) and (4), two trigonometric functions plus one division are replaced by two additions and two multiplications.

The phase detector of the 2S-PLL [3] is based on the Park transformation. The phase error signal, i.e. the $q$ component, is evaluated from the grid voltage phasor projections on the rotating reference frame synchronized with the PLL phase. In order to reduce the computational burden, while retaining the required accuracy at a low sampling rate, it is proposed to replace the trigonometric functions required for the Park transformation with a digital oscillator [4]: 


$$
\begin{gathered}
(\sin \theta)_{k}=A_{2}(\cos \theta)_{k-1}+\left(1+A_{1} A_{2}\right)(\sin \theta)_{k-1} \\
(\cos \theta)_{k}=(\cos \theta)_{k-1}-A_{1}\left((\sin \theta)_{k-1}+(\sin \theta)_{k}\right)
\end{gathered}
$$

where $A_{1}=\tan \left(\frac{\pi}{N}\right)$ and $A_{2}=\sin \left(\frac{2 \pi}{N}\right)$.

If required, the computational burden associated to the normalization block of the PLL can be minimized by replacing the associated operations with a gain, whose value depends on the nominal grid voltage. With all the above proposed approximations, the 2S-PLL can be implemented, following the block diagram in Fig. 1, using sequentially only one addition/subtraction and one multiplication elements, given that $T_{s}$ is large enough.

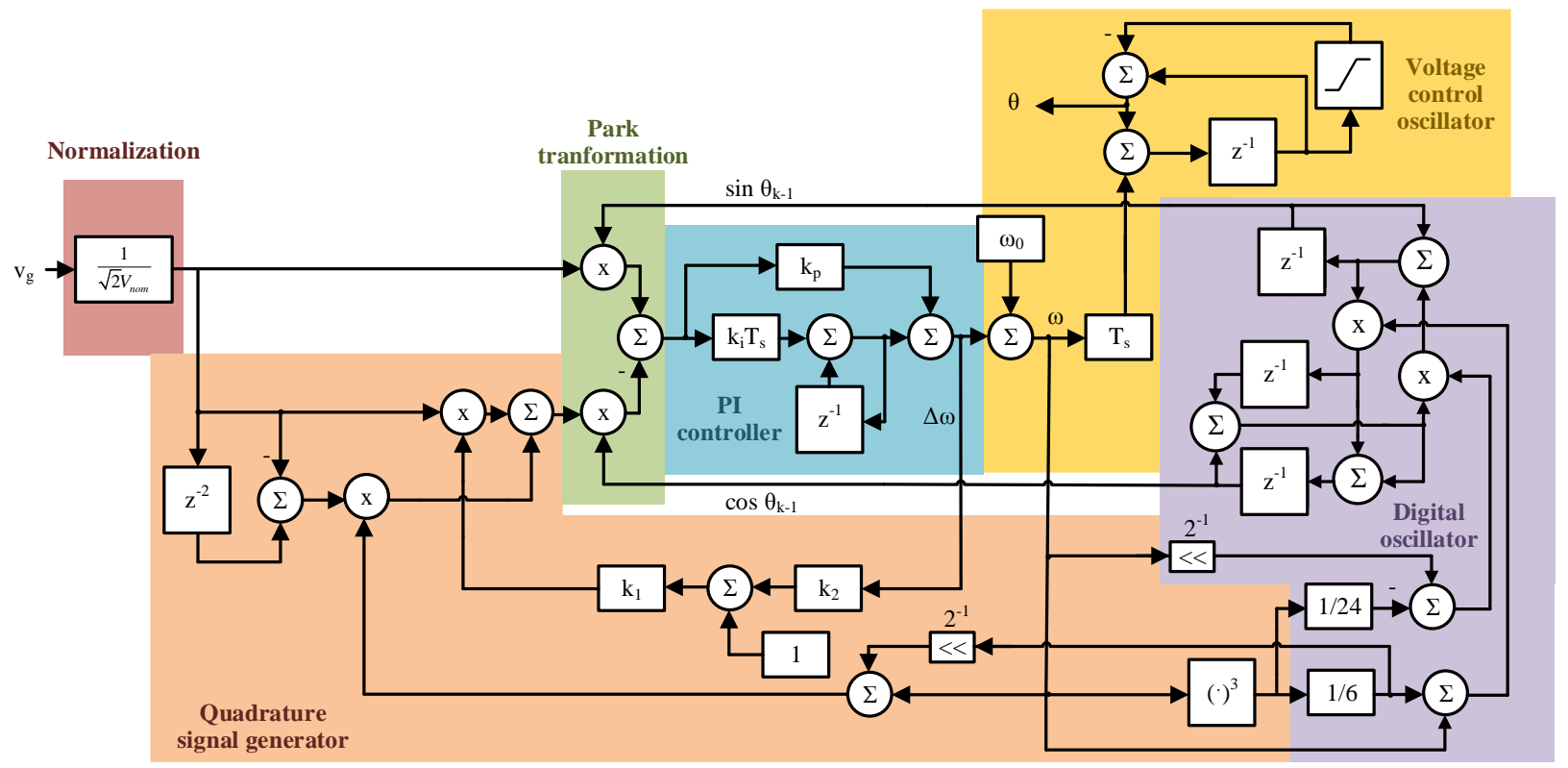

Fig. 1. Proposed 2S-PLL.

\section{SIMULATION RESULTS}

The original and proposed 2S-PLL are evaluated by simulation and their performances compared with the T/4 PLL one. These PLLs have been designed with the same PI controller parameters, according to [5] (proportional, $K_{p}=46$ and integral gain, $K_{i}=1024$ ). To obtain a first proof of concept, initial results are presented using the lowest sample rate in which the PLL obtains a response similar to the original $2 \mathrm{~S}$, demonstrating that the sample rate can be comfortably 
adjusted to the switching frequency. The nominal frequency has been set to $50 \mathrm{~Hz}$ and $T_{s}$ is $1 / 800 \mathrm{~s}$.

The responses to a $+10 \mathrm{~Hz}$ frequency step are shown in Fig. 2. The phase errors due to both $2 \mathrm{~S}-$ PLL matches and are below the T/4 one in steady-state. The final manuscript will provide a detailed comparison in simulation.

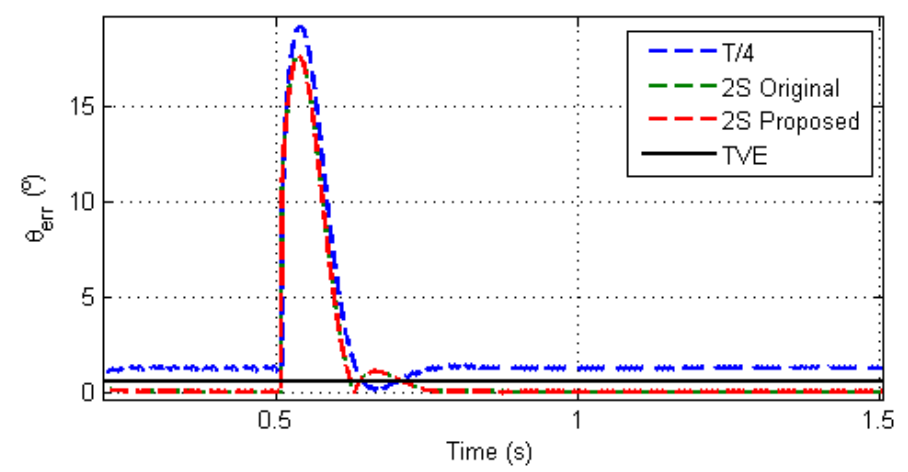

Fig. 2. Phase error due to a frequency step from 45 to $55 \mathrm{~Hz}$.

\section{EXPERIMENTAL RESULTS}

The proposed PLL has been implemented in a FPGA to assess the size of the circuits and synchronization capabilities. Table I summarizes the resources used by the proposed PLL in an Artix 7 FPGA (XC7A100T-1CSG324C, Nexys 4 board).

Table 1: Summary of the FPGA resources used by the PLLs using SysGen.

\begin{tabular}{|c|c|c|c|c|c|c|c|}
\hline & $\begin{array}{c}\text { Slice } \\
\text { Registers }\end{array}$ & LUTs & $\begin{array}{c}\text { Occupied } \\
\text { Slices }\end{array}$ & $\begin{array}{c}\text { LUT Flip Flop } \\
\text { pairs used }\end{array}$ & $\begin{array}{c}\text { RAMB/ } \\
\text { FIFOs }\end{array}$ & $\begin{array}{c}\text { BUFG/ } \\
\text { BUGCTROLs }\end{array}$ & DSP48E1s \\
\hline T/4 PLL & 3472 & 4927 & 1697 & 5144 & 3 & 1 & 64 \\
\hline $\begin{array}{c}\text { 2S-PLL } \\
\text { ORIGINAL }\end{array}$ & 3488 & 5401 & 1746 & 5563 & 2 & 1 & 72 \\
\hline $\begin{array}{c}\text { 2S-PLL } \\
\text { PROPOSED }\end{array}$ & 238 & 1940 & 662 & 2033 & 0 & 1 & 32 \\
\hline
\end{tabular}

In Fig. 3, the grid voltage, frequency and phase error signals are shown when a $+6 \mathrm{~Hz}$ frequency step occurs, proving the effectiveness of the proposed PLL. In the final version, more results will be provided. 




(a)

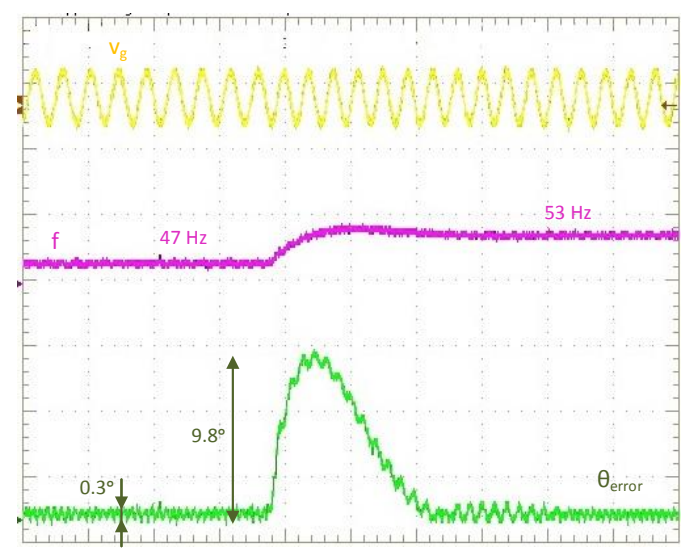

(b)

Fig. 3. Grid voltage (yellow), frequency (green) and phase error (magenta) waveforms using proposed $2 S$-PLL under $+6 \mathrm{~Hz}$ frequency step from 47 to $53 \mathrm{~Hz}$. (a) T/4 and (b) $2 S$ PLL.

\section{CONCLUSIONS AND FUTURE WORK}

A novel approach in digital implementation of 2S-PLL applied for low switching frequency to single-phase PFC has been presented in this work. The proposed methodology mainly focused on low cost implementation that allows a reduction of up to $65 \%$ of the resources used in the FPGA with respect to the original 2S-PLL. Based on this approach, the PLL has been implemented, tested and verified that the phase error that occurs with the proposed PLL is lower, both at steady state and under frequency variations, than that obtained with one of the simplest strategies found in the literature, the T/4-PLL.

\section{REFERENCES}

[1] F. López, V. M. López-Martín, F. J. Azcondo, L. Corradini, and A. Pigazo, "Current-Sensorless Power Factor Correction with Predictive Controllers," IEEE J. Emerg. Sel. Top. Power Electron., vol. 7, no. 2, pp. 891-900, Jun. 2019, doi: 10.1109/JESTPE.2019.2896768.

[2] L. Huber, B. T. Irving, and M. M. Jovanovic, "Review and Stability Analysis of PLL-Based Interleaving Control of DCM/CCM Boundary Boost PFC Converters," IEEE Trans. Power Electron., vol. 24, no. 8, pp. 1992-1999, Aug. 2009, doi: 10.1109/TPEL.2009.2018560.

[3] P. Lamo, F. López, A. Pigazo, and F. J. Azcondo, "An Efficient FPGA Implementation of a Quadrature Signal-Generation Subsystem in SRF PLLs in Single-Phase PFCs," IEEE Trans. Power Electron., vol. 32, no. 5, pp. 3959-3969, May 2017, doi: 10.1109/TPEL.2016.2582534.

[4] M. Vicanek, "A New Recursive Quadrature Oscillator," 21-Oct-2015. [Online]. Available: https://vicanek.de/articles/QuadOsc.pdf.

[5] R. Teodorescu, M. Liserre, and P. Rodriguez, Grid converters for photovoltaic and wind power systems, vol. 29. John Wiley \& Sons, 2011. 